\title{
Gobernanza ambiental para el desarrollo sostenible de la cuenca de Santiaguillo, Durango
}

\section{Environmental governance for sustainable development of Santiaguillo's basin, Durango}

doi: http://dx.doi.org/10.32870/

espiral.v25i72.6038

\section{Resumen}

Este artículo presenta los resultados de un proyecto de investigación que tuvo como objetivos generar e implementar una estrategia de gobernanza ambiental para encaminar acciones para el uso sustentable de los recursos hídricos de la cuenca de Santiaguillo, en el estado de Durango, México. Con la metodología de investigación-acción participativa, y con base en un marco teórico-conceptual, se identificaron actores sociales clave y se llevaron a cabo acciones. Se concluye que a través de la gobernanza ambiental se incentivan acciones que encaminan al uso sustentable de los recursos hídricos de la cuenca de Santiaguillo, como el ordenamiento intermunicipal de la cuenca y la participación activa de la sociedad civil, organizaciones no gubernamentales (ONG), empresas y Gobierno.

Palabras clave: gobernanza ambiental, desarrollo sostenible, cuenca, sociedad civil, Durango.

\author{
Erika Cassio Madrazo* \\ Eduardo Sánchez Ortiz ${ }^{\star \star}$
}

\begin{abstract}
This article presents the results of the research project, which objectives were to generate and implement an environmental governance strategy that directs actions for the sustainable use of the water resources of the Santiaguillo's basin. Through the methodology of participatory action research, and based on the theoreticalconceptual framework, key social actors were identified and actions were taken. It is concluded that -through environmental governance, actions are encouraged that lead to the sustainable use of the water resources of the Santiaguillo's basin, such as the intermunicipal order of the basin, and the active participation of civil society, NGOs, companies and government.
\end{abstract}

Keywords: Environmental governance, sustainable development, basin, civil society, Durango.

- Profesora-Investigadora el Centro Interdisciplinario de Investigación para el Desarrollo Integral (CIIDIR), unidad Durango, del Instituto Politécnico Nacional (IPN), México. ORCID: http://orcid.org/0000-0003-2703-9063_erikassio@gmail.com

- Profesor-Investigador en la Academia de Gestión Territorial del Centro Interdisciplinario de Investigación para el Desarrollo Integral (CIIDIR), unidad Durango, del Instituto Politécnico Nacional (IPN), México. ORCID: http://orcid.org/0000-0002-I88I-5537_esanchezo@ipn.mx Fecha de recepción: 22 de agosto de 2016. Fecha de aceptación:09 de febrero de 2018. 


\section{Introducción}

A partir de los años ochenta, se popularizo entre los académicos y organismos no gubernamentales, como la Organización de las Naciones Unidas (ONU), el paradigma de que el desarrollo tenía que ser sostenible o sustentable, esto es, satisfacer las necesidades de las generaciones presentes sin comprometer la capacidad de las generaciones futuras para satisfacer sus propias necesidades (Comisión Mundial sobre el Medio Ambiente y el Desarrollo, 1987). Dicho modelo de desarrollo implica políticas, instituciones, estilos de vida y formas de producción, consumo, distribución y movilidad más racionales en términos ecológicos, económicos y sociales, además de más éticos (Jiménez, 2003).

Esta propuesta surgió a partir de que la ciencia comenzó a demostrar la existencia de deterioros en: 1) el medio ambiente natural, del cual dependemos completamente como especie, a través de fenómenos como el cambio climático y la contaminación atmosférica del agua y de los alimentos; 2) el medio social, resultando en desnutrición, hambre y violencia; y 3) la economía, que se refleja en procesos de inflación sin control, desempleo y distribución extremadamente desigual del ingreso y la riqueza (Costanza, Cumberland, Daly, Goodland y Norgaard, 1999).

Actualmente, se dispone, sobre todo en los países desarrollados y gracias a la tecnología como el internet y los medios de comunicación, de una gran cantidad de información que ayuda a vencer la ilusión de independencia de los seres humanos respecto al medio ambiente y el sistema económico (Espinoza, 2004). Esto ha provocado que las personas tomen conciencia sobre las limitaciones del orden mundial y del control público como mecanismo de gobierno, y también sobre las formas como todos hemos respondido a los problemas sociales y ambientales que requieren un mayor número de enfoques, instrumentos y acciones (Kooiman, 2004). 
Debido a lo anterior, Kooiman (2004) establece que la sociedad en gran parte del mundo ha generado acciones a través de la organización y conformación de asociaciones civiles llamadas organizaciones no gubernamentales (ONG). Estas ONG actúan en asuntos humanitarios de distinta índole, buscando hacer frente a la injusticia, corrupción, pobreza, deterioro ambiental, la inequidad y el abuso de poder, entre otros problemas.

La emergencia de estos grupos ha propiciado cambios significativos en los procesos de toma de decisiones y en la forma de gobernar, esto derivado de su exigencia de información y de su involucramiento en la gestión de organizaciones públicas y privadas, así como por su participación en manifestaciones civiles pacíficas o violentas, que han propiciado en alguna medida acciones colectivas encaminadas a mitigar las consecuencias negativas de los deterioros antes mencionados (Kooiman, 2004).

Existen organizaciones de todo tipo que, de acuerdo con Dúran (2009), se clasificarían en tres sectores principalmente: el primero estaría representado por el Gobierno o el Estado, el segundo por el mercado, y el tercero por la sociedad civil. Estos tres sectores ejercen diferentes grados de poder y presión en la toma de decisiones relacionadas con el planeta, y estos van acorde con sus intereses, propósitos, principios y valores.

Por sus fines y propósitos, las organizaciones del tercer sector están orientadas al bienestar social y ambiental y buscan dar voz a los que no la tienen, como la flora y la fauna, así como a quienes, aunque la tengan, no son escuchados, como los grupos sociales oprimidos, pobres, violentados, enfermos, marginados, etc. (García, 2008; Dúran, 2009; Vélez, 2011).

La sustentabilidad implica el equilibrio entre los sistemas humanos (económico, social, político) y ambientales, y ha sido asociada a la gobernanza desde diferentes visiones 
y niveles (Jiménez, 2003). En particular, la gobernanza ambiental analiza las interrelaciones de los diferentes actores (en este caso organizaciones del primer, segundo y tercer sector), y la dinámica de la toma de decisiones para el manejo eficiente y eficaz de los ecosistemas.

Por otra parte, las cuencas hidrológicas o hidrográficas son espacios considerados unidades territoriales básicas para la planeación, gestión y manejo sustentable de los recursos naturales, además de dimensiones espacio-temporales para la adaptación ante el cambio climático, esto debido a que, según Ávalos, Alcántar, Mora, López y Patrón (2013), los principales impactos del cambio climático ocurren en todos aquellos aspectos relacionados con el agua, como el aporte de la misma para actividades productivas y de consumo humano, o eventos como sequías e inundaciones, entre otros.

La cuenca de la Laguna de Santiaguillo, ubicada en el estado de Durango, presenta algunos problemas sociales y ambientales. Siguiendo las aportaciones de los autores en relación con el Gobierno de los bienes comunes, las teorías de cooperación, acción colectiva y gobernanza ambiental, y desde un enfoque de manejo integral y sustentable de cuencas, el presente trabajo se planteó el objetivo de determinar si la acción colectiva a través de la conformación de una asociación civil integrada por usuarios y líderes de la cuenca de Santiaguillo promovería la gobernanza ambiental y encaminaría acciones para el uso sustentable de los recursos hídricos en aquella.

En el primer apartado, se definen los conceptos manejo integral de cuencas, gobernanza ambiental y acción colectiva, que fueron utilizados para el estudio de la problemática que se presenta en la cuenca de Santiaguillo. En el segundo apartado, se determina cuál es la problemática de dicha cuenca. En el tercero, se establece cómo fue el proceso metodológico que se llevó a cabo para enfrentar dicha pro- 
blemática desde la investigación-acción. Finalmente, en el cuarto apartado se presentan los resultados.

\section{Manejo integral de cuencas, gobernanza ambiental y acción colectiva}

Se considera que una cuenca hidrológica es todo aquel espacio delimitado por áreas divisorias de sistemas de arroyos, ríos y partes altas de montañas, que escurren, confluyen y desembocan en un punto común llamado también punto de salida de la cuenca, que puede ser un lago, formando una cuenca llamada endorreica, o el mar, llamada cuenca exorreica (Donzier, Walshe, Brühl, Cordeiro, Estrela, et al., 2009; Ávalos, Alcántar, Mora, López y Patrón, 2013).

Son unidades sumamente complejas:

En estos territorios hay una interrelación e interdependencia espacial y temporal entre el medio biofísico (suelos, ecosistemas acuáticos y terrestres, cultivos, agua, biodiversidad, estructura geomorfológica y geológica), los modos de apropiación (tecnología y/o mercados) y las instituciones (organización social, cultura, reglas y/o leyes) (Ávalos, Alcántar, Mora, López y Patrón, 2013, p. 7).

Las cuencas hidrológicas se pueden clasificar en subterráneas y superficiales, y su manejo se considera un proceso complejo que da orden a un conjunto de acciones que buscan lograr desarrollo social y económico sostenibles en el tiempo, además de la protección del medio ambiente (Piedra, 2007). La disponibilidad de agua es un factor determinante para el desarrollo de comunidades humanas, por lo que las cuencas se consideran unidades de paisaje para el manejo integral de los recursos hídricos (Maass y Cotler, 2007).

El manejo integral de cuencas se considera un proceso que busca la solución de problemas complejos interrelacionados, el cual debe ser adaptativo, esto es, que se aprende 
y construye con base en las experiencias y con sustento en información científica y local. Es un proceso que requiere la concurrencia, la cooperación, la colaboración y la acción colectiva de los actores e instituciones involucrados en la toma de decisiones (Ávalos, Alcántar, Mora, López y Patrón, 2013).

Para Ávalos, Alcántar, Mora, López y Patrón (2013), este proceso debe ser flexible y adaptativo, a través de cuatro fases o etapas: 1) diagnóstico/identificación del problema; 2) planeación (áreas prioritarias, acciones, involucramiento de la población); 3) implementación (prácticas: ¿qué mejoras se esperan con ellas?); y 4) evaluación (¿los resultados son los esperados o son necesarios algunos cambios?).

Maass y Cotler (2007) proponen una serie de pasos a seguir para el manejo integral de cuencas que implica el diagnóstico y síntesis de la información, la definición del ecosistema, la definición de objetivos y metas, la construcción de escenarios, el diseño de intervenciones, la implementación del manejo, el monitoreo, evaluación y seguimiento, y, por último, la retroalimentación y el manejo adaptativo.

Figura 1. Pasos a seguir en el manejo integrado de cuencas hidrográficas

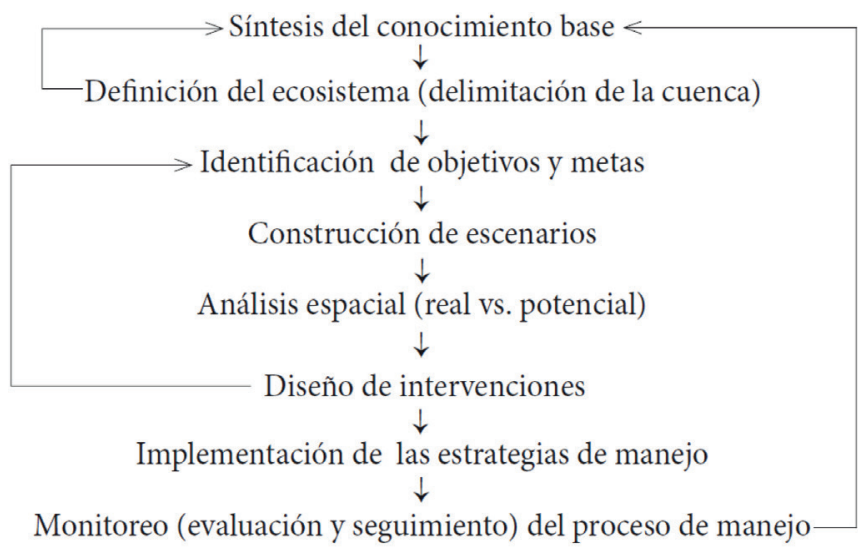

Fuente: Maas y Cotler (2007, p. 43). 
El proceso para el manejo integral de cuencas propuesto por diferentes autores implica la acción colectiva, entendida como "aquellas actividades que requieren del esfuerzo coordinado de dos o más individuos” (Moya, 2004, p. 108), y actividades relacionadas con la gestión ${ }^{1}$ como planeación, implementación y evaluación de acciones (Ávalos, Alcántar, Mora, López y Patrón, 2013). Para Ávalos, Alcántar, Mora, López y Patrón (2013), este proceso se inicia cuando los habitantes se reconocen como parte de la cuenca y como beneficiarios de sus servicios ecosistémicos.

Cotler (2007) establece que el manejo integral de cuencas parte del supuesto básico de que los actores tendrán la capacidad de coordinarse en torno a un objetivo común, en este caso la conservación de su entorno y el sostenimiento de sus actividades productivas, ya que las cuencas son zonas donde existen recursos de uso común, principalmente el agua: "La implementación de un manejo integral de cuencas requiere la concurrencia, la cooperación y la colaboración de diversas instituciones bajo una visión común” (Cotler, 2007, p. 13).

En 1965, Mancur Olson sentó las bases de la teoría de la acción colectiva, la cual supone que las personas o individuos deciden su participación en acciones colectivas a partir de calcular los costos y beneficios que obtendrían, desde un enfoque economista. Por otro lado, hay autores como Granovetter (1978), que consideran que existen otras razones por las que los actores deciden participar en acciones colectivas, como el número de participantes en ellas (Moya, 2004).

La acción colectiva está relacionada con la decisión de cada actor de cooperar o no para el cumplimiento de objetivos y metas, lo cual puede estar determinado por factores como la confianza, el beneficio individual y colectivo, las relaciones entre actores, la reputación de los individuos y la solidaridad, entre otros (Vargas, 2003; Moya, 2004; Nowak, 2006). 
A pesar de que autores como Hardin (1968) aseguran que la estrategia dominante ante la escasez de algún recurso común es la de no cooperar y seguir cada actor su propio interés, precipitándose hacia el gorroneo o free-ride (Hardin, 1968), autores como Elionor Ostrom (2000) aseguran que esto no es una regla.

Ostrom (2000) señala que en una situación de interdependencia relacionada con el uso de recursos comunes, los actores pueden organizarse y gobernarse a sí mismos para obtener beneficios conjuntos a pesar de la tentación del gorroneo, esto por varios elementos: los costos/beneficios de las acciones de los individuos y de su vinculación con los resultados esperados; la reducción de incertidumbre causada por la falta de conocimiento sobre el sistema de recursos explotables; un proceso de aprendizaje basado en ensayo y error; y la provisión y apropiación de los recursos de uso común.

Ahora bien, se considera que existen actores del sector público y privado para el manejo integral de cuencas. Para Zárate (2007), en México el manejo y gestión de las cuencas, al igual que en otros países, sigue estando a cargo del Gobierno, especialmente del federal. Este autor considera que la transición de gobierno a gobernanza o gobernabilidad de los recursos hídricos ha sido lenta y difícil.

Ahora estamos en el camino de construir sistemas de gestión en los que, en una visión idealizada a largo plazo, los Gobiernos de los distintos niveles se coordinan entre sí; los ciudadanos intervienen organizadamente en las decisiones que les competen y les afectan; las decisiones se toman lo más cercanamente posible a los lugares en que se generan los problemas; se dispone de información completa y esta se encuentra a disposición de todos los interesados; la planificación es ordenada, sistemática y participativa; [y] las intervenciones de las entidades gubernamentales $y$ las empresas cuidan los impactos y mitigan los efectos indeseables de las actividades productivas $y$ el desarrollo urbano, $y$ teniendo presentes 
las múltiples relaciones e interrelaciones que se producen entre los recursos naturales, la biodiversidad y los ecosistemas $y$, todo ello, en el marco geográfico de las cuencas hidrológicas (Zárate, 2007, p. 188).

Para Delgado, Bachmann y Oñate (2007), la gobernanza ambiental se refiere a los procesos de toma de decisiones en relación con bienes públicos en los que intervienen el Estado, las empresas y la sociedad civil, que tienen que ver con el establecimiento de marcos regulatorios para la conservación, los límites y las restricciones sobre el uso de los recursos naturales y los ecosistemas. El enfoque se basa en la idea de que la gestión de los servicios ecosistémicos es ya no función exclusiva del Estado, sino también de los actores interesados que ejercen un control, propiedad y hacen uso de ellos (Delgado, Bachmann y Oñate, 2007).

Para Zárate (2007), el propósito parece claro, pero no la estrategia y el cómo lograrlo. Kooiman (2004) considera que el control público como mecanismo de gobierno posee limitaciones, y que es importante incluir nuevas formas de actuar y enfrentar los problemas sociales y ambientales, esto es, diferentes enfoques provistos por los diferentes actores.

La sociedad civil se ha organizado en forma política ${ }^{2}$ fuera del Estado. Es una forma de organización de la sociedad autorregulada, descentralizada y voluntaria, basada en una vida moral del Estado y organizada de forma autónoma. La función central de las conductas y prácticas de estas organizaciones de la sociedad civil, también conocidas como ONG, se fundamenta en relaciones interdependientes de confianza mutua, reciprocidad e intercambio (Vargas, 2003).

Así, en el caso concreto del presente trabajo, con el fin de lograr el manejo y gestión integrada de las cuencas, se

2. La política es una actividad orientada en forma ideológica a la toma de decisiones de un grupo para alcanzar ciertos objetivos. También puede definirse como una manera de ejercer el poder con la intención de resolver o minimizar el choque entre los intereses encontrados que se producen dentro de una sociedad. 
han conformado algunas figuras de participación y toma de decisiones promovidas por la sociedad civil y la academia como consejos, comités y comisiones, integrados por actores interesados en encaminar acciones hacia un manejo sustentable de los recursos comunes de las cuencas, especialmente los hídricos (Ávalos, Alcántar, Mora, López y Patrón, 2013).

\section{La cuenca de Santiaguillo en Durango, México}

La cuenca endorreica de Santiaguillo se encuentra en el estado de Durango, México, dentro de la cuenca San Pedro-Mezquital, con una extensión de 2500 km² en donde realizan sus actividades poco más de 30000 habitantes distribuidos en ciento cincuenta y tres localidades, principalmente rurales. Abarca políticamente cinco municipios, de los cuales destacan por su extensión Canatlán, Nuevo Ideal y Santiago Papasquiaro. Estas localidades emplean para sus actividades -agricultura, ganadería, comercio y fruticultura- un total de ciento cuarenta y nueve aprovechamientos superficiales y 1964 aprovechamientos subterráneos (Montenegro, 2014) (Figura 2).

De la superficie total de la cuenca, se dedican a la agricultura 110184 ha (43.35\%), de las cuales el 84.4\% (90 819 ha) es de temporal y el 17.6\% (19 320 ha) de riego. Sin agricultura, existen 144075.2 ha que corresponden al 56.7\% de la superficie total, y en las que es posible identificar un humedal potencial que cubre 24684.4 ha, superficie de pastizales de 41952.9 ha, y superficie de matorrales de 10018.7 ha (6.9\%). El aprovechamiento de aguas subterráneas se realiza a través de 1228 pozos, siendo los mayores consumidores los productores del sector agrícola, con un $86 \%$ del volumen total extraído, seguidos por el uso público urbano, con el $12 \%$ (Montenegro, 2014). 
Figura 2. Mapa de ubicación de la cuenca de Santiaguillo

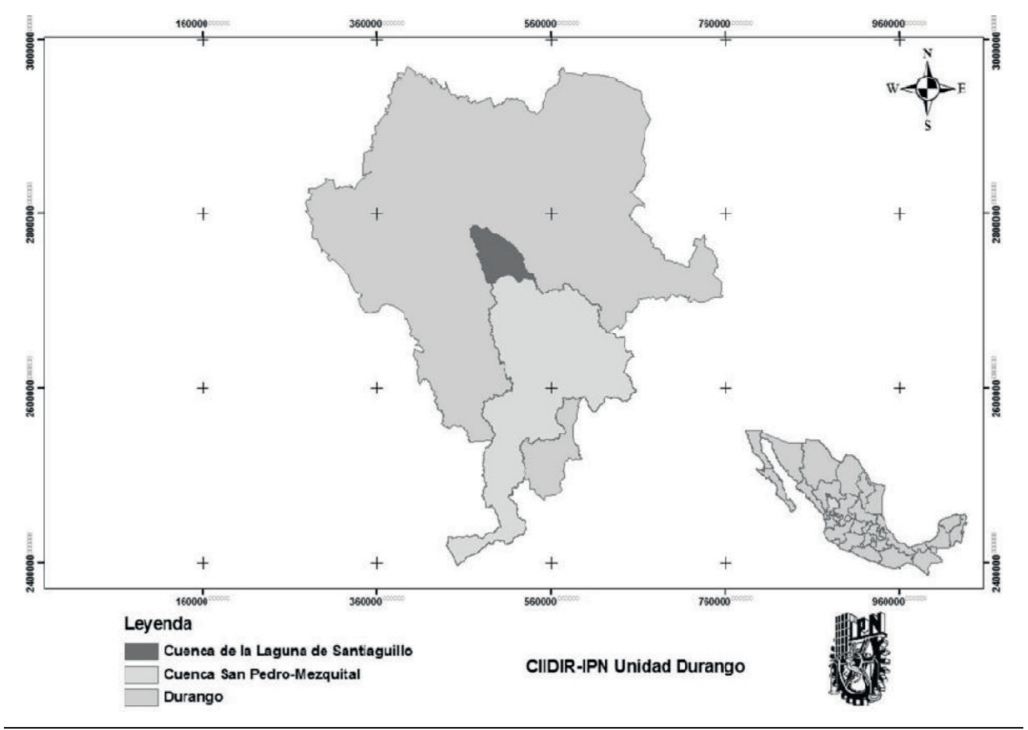

Fuente: Montenegro (2014).

A pesar de que la Laguna de Santiaguillo se considera una fuente de agua importante que alberga sesenta y siete especies de aves migratorias, y es clasificada como humedal-sitio Ramsar y como área de importancia para conservación de aves (AICA), estudios recientes establecieron que existe una sobreexplotación del 300\% de su capacidad de recarga, de acuerdo al volumen concesionado por la Comisión Nacional del Agua (CONAGUA), contra la capacidad de recarga total media anual del acuífero.

La concesión de volúmenes de agua es un derecho tutelado por la federación, por lo que la solución de esta problemática no tiene vías fáciles a partir de la aplicación de las atribuciones del Estado, quien vulnera el Registro Público de Derechos de Agua mediante acciones arbitrarias de ejercicio del poder. Por tanto, las soluciones accesibles corresponden al diagnóstico, sensibilización y la construc- 
ción de acuerdos locales en la sociedad encaminados a la protección del acuífero y la mejora de la eficiencia del uso de las aguas superficiales y subterráneas. Por tanto, es necesario fortalecer las capacidades sociales de autogestión a fin de establecer condiciones de desarrollo sustentable y preservación ambiental, esto mediante soluciones locales.

La sobreexplotación del recurso hídrico de la cuenca es sólo uno de los problemas de importancia que presentan las cuencas en México: la mayor parte de ellas manifiestan impactos acumulados por las actividades humanas en cuanto al aporte de minerales, sedimentos y nutrientes, un aporte de compuestos organoclorados y metales pesados, de sedimentos, de bacterias y basura, así como ríos eutrofizados y contaminados, entre otros, todo lo cual provoca serios problemas sociales y económicos.

Además, la Organización Internacional para las Migraciones, en el 2011, estableció que la degradación de los ecosistemas provoca la migración de las especies, incluidas la humana, lo que amenaza la sustentabilidad de los territorios. En la cuenca de Santiaguillo, de 1990 al 2010 se presentó una reducción poblacional del $0.66 \%$ por dicho fenómeno.

De acuerdo al marco teórico-conceptual, y considerando el poder que puede llegar a adquirir la sociedad civil organizada a través de una coordinación formal orientada hacia la toma de decisiones que estén encaminadas al manejo integral y sustentable de los recursos hídricos de la cuenca, se planteó realizar un proyecto de investigación en donde los actores sociales representaran no sólo objetos de estudio, sino también agentes de cambio. Debido a esto, se decidió trabajar bajo una metodología que permitiera el involucramiento activo del grupo de investigación con la sociedad. A continuación, se describe dicha metodología implementada. 


\section{Proceso metodológico}

De acuerdo al marco teórico-conceptual, y habiendo establecido dentro del objetivo de la investigación realizada la conformación de una asociación civil, se determinó utilizar la metodología investigación-acción participativa (IAP), la cual se basa en dos procesos básicos: el conocer y el actuar (Kirchner, 2007).

"La investigación-acción participativa ha sido conceptualizada como un proceso por el cual miembros de un grupo o una comunidad oprimida colectan y analizan información y actúan sobre sus problemas con el propósito de encontrarles soluciones y promover transformaciones políticas y sociales" (Balcazar, 2003, p. 6), esto facilitado por agentes externos y basados en una metodología. Se basa en la inserción del investigador en la comunidad, el cual analiza las condiciones históricas y la estructura social de la misma, además de contribuir o facilitar el desarrollo del nivel de conciencia de los miembros de la comunidad, el desarrollo de organizaciones políticas, así como de grupos de acción (Balcazar, 2003).

Esta metodología considera que las personas que participan tienen la capacidad de contribuir de forma activa a la investigación -esto independientemente de su grado de escolaridad-, que cada persona es valiosa, y que todas son capaces de transformar su realidad social, siempre y cuando exista en ellas deseo y voluntad (Balcazar, 2003).

La IAP parte de la idea de que existen grupos vulnerables y minorías que son oprimidas o dominadas por los grupos de poder, y que esa misma historia de explotación y alienación no les permite tomar la iniciativa para transformar su realidad, por lo que agentes externos como los grupos de investigación pueden insertarse en las comunidades como detonantes de cambio y guías en el proceso de transformación. La IAP también parte de la creencia de que la investigación social debe aportar a la disminución de la injusticia 
en la sociedad, promover la participación de los miembros de las comunidades en la búsqueda de soluciones a sus propios problemas, y ayudar a los miembros de las comunidades a incrementar el grado de control que ellos tienen sobre aspectos relevantes de sus vidas, esto es, incrementar su poder en la toma de decisiones (Pereda, Prada y Actis, 1993; Balcazar, 2003).

La IAP considera diferentes etapas dentro del proceso de investigación y acción. Estas varían según el autor, pero para este estudio se consideraron cuatro fases o etapas para el logro de los diferentes objetivos: investigación, educación, acción y cierre. Este proceso puede ser cíclico, ya que se puede cumplir con diferentes objetivos y comenzar de nuevo el proceso con el fin de dar continuidad a las acciones (Figura 3).

Figura 3. Proceso de IAP

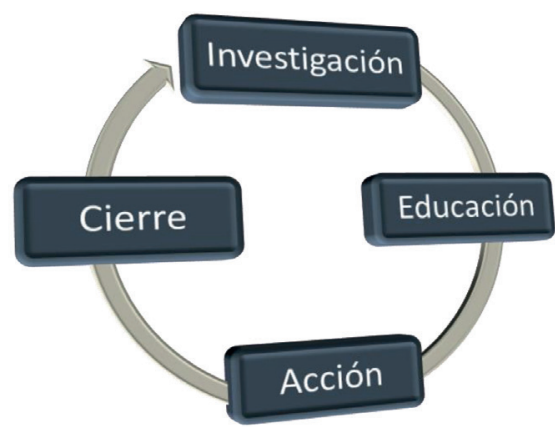

Fuente: elaboración propia basada en Balcazar (2007).

En cada una de las etapas señaladas se establecieron objetivos y se realizaron acciones para cumplirlos. En las Tablas 1, 2, 3 y 4 se muestran las etapas del proceso IAP, los objetivos y las actividades realizadas. 


\section{Tabla 1. Objetivos y acciones en la etapa de investigación}

Objetivos específicos

Analizar las condiciones históricas de la cuenca a través de la participación de los pobladores

Analizar de forma sistémica las condiciones actuales de la problemática

Identificación de necesidades o preocupaciones de la población de forma participativa

Determinar prioridades y organizar grupos de acción para planear de forma sistemática el proceso de solución de los problemas de forma participativa

\section{Acciones}

Se identificaron actores de mayor edad, productores y líderes dentro de la cuenca en las diferentes localidades y se realizaron entrevistas videograbadas. Con esto se elaboró un cortometraje denominado Saberes, que fue utilizado como herramienta para la sensibilización en talleres y grupos focales.

Se hicieron cinco transectos con guía de observación y entrevista semiestructurada a los comisariados ejidales y productores, cubriendo las localidades que representan el $90 \%$ de la población y el $100 \%$ de los tamaños de localidad en la cuenca.

Se realizaron talleres de diagnóstico participativo y se utilizaron herramientas como árbol de ideas, cuadro comparativo, lluvia de ideas y foros de discusión.

Curso-taller de cartografía para la identificación de las microcuencas y sensibilización de interrelaciones entre pobladores.

Identificación de líderes y actores comprometidos a participar en la asociación civil para la conformación de grupos de acción por microcuencas y elección de representantes.

Fuente: elaboración propia con base en diseño de investigación. 


\section{Tabla 2. Objetivos y acciones en la etapa de educación}

Objetivos específicos

Generar acciones que permitan a los participantes aprender a desarrollar una conciencia crítica

Generar acciones que permitan a los participantes identificar las causas de sus problemas

Generar acciones que permitan a los participantes descubrir su propio potencial para actuar

\section{Acciones}

Taller Apropiación del concepto de cuenca. Curso de aplicación de sistemas de información geográfica (SIG) en análisis territorial.

Taller de elaboración del reglamento interno y agenda de trabajo.

Curso-taller de cartografía del medio físico: cuerpos de agua, tipos de clima, cobertura del suelo, hidrología, elevaciones y precipitación.

Taller de educación ambiental dirigido a profesores de primaria y secundaria. Curso sobre pastizales dirigido a técnicos agropecuarios.

Taller de género dirigido a la asociación de pescadoras, jóvenes mexicanos y menonitas.

Talleres de educación ambiental, género y agua.

Taller de integración del plan de gestión integrada.

Fuente: elaboración propia con base en diseño de investigación. 


\section{Tabla 3. Objetivos y acciones en la etapa de acción}

\section{Objetivos específicos}

Acciones

Logar que los partici- Integración de un comité representativo de la poblapantes implementen ción de la cuenca.

soluciones prácticas a sus problemas, utilizando sus propios Conformación de tres unidades piloto en diferentes comunidades para probar técnicas de manejo agrícola sustentables.

recursos o en solidari- Gestión por parte del comité con los tres niveles de dad con otros grupos Gobierno para la implementación de un programa o gremios a través de acciones interrelacionadas y en un ciclo dinámico de ordenamiento.

Gestión de apoyo por parte del comité ante asociación Pronatura A. C., para financiamiento para realizar acciones de evaluación y mitigación de impacto ambiental.

Fuente: elaboración propia con base en diseño de investigación.

\section{Tabla 4. Objetivos y acciones en la etapa de cierre}

\section{Objetivos específicos}

Permitir que los participantes y actores Formalización el comité como asoinvolucrados en el manejo sustentable ciación civil ante notario público. de la cuenca lleven a cabo las acciones Creación de reglamento interno. necesarias de manera autónoma, esto Creación de organigrama interno. es, sin el liderazgo del grupo de investi- Generación de un programa de gación y a través de sus propias capaci- acciones a corto, mediano y largo dades de autogestión. plazo.

Permitir que el comité controle su Retirada de la toma de decisiopropia agenda, adquiera el compromiso nes por parte del grupo de invesnecesario para apropiarse de la organi- tigación. zación y genere las acciones necesarias que lo encaminen a cumplir con su propósito.

Fuente: elaboración propia con base en diseño de investigación. 


\section{Resultados}

Los resultados obtenidos en las diferentes etapas se describen a continuación.

\section{I. Etapa de investigación}

En la etapa de investigación, se realizó una caracterización social de la cuenca, en la cual se establecieron la demografía, la pirámide poblacional, la estratificación socioeconómica y la identificación de etnias y grupos sociales. De los aspectos relevantes que se encontraron, se destaca que la mayoría de las personas en la región se encuentran asociadas en organizaciones ejidales para la realización de actividades productivas y la toma de decisiones que atañen a los ejidos como territorio. La forma organizacional ejidal es de las formas de organización más importantes y representativas en la región de la cuenca: la mayor parte de los productores trabaja dentro de dicha forma organizacional. Por otro lado, se encontró que la mayor parte de los pobladores se encuentra en un rango de edad de entre treinta y sesenta años, y los liderazgos se ubican en adultos de cincuenta años de edad en promedio. En las entrevistas que se videograbaron con los adultos mayores de sesenta años que nacieron y crecieron en la región de la cuenca, se denotó el conocimiento que poseen los habitantes en cuanto a la degradación de los recursos hídricos y las posibles consecuencias de su deterioro. Por otro lado, se demostró que la mayor parte del territorio se utiliza para actividades productivas, por lo que los actores que más inciden en el manejo de la cuenca son los productores, principalmente los agricultores.

Para el manejo integral y sustentable de las cuencas, resulta importante que los pobladores se sientan identificados con su territorio y tengan conocimiento de él. Por otro lado, para el grupo de investigación resulta importante 
comprender la percepción que se tiene por parte de los habitantes acerca de su propio territorio.

Debido a lo anterior, el medio físico se investigó a través de métodos participativos y utilizando sistemas de información geográfica. Los participantes identificaron los cuerpos de agua, los tipos de clima, la cobertura del suelo, la hidrología, las elevaciones y las precipitaciones de su territorio. Así, se logró generar una descripción -textual y mediante mapas- del balance hidrológico a nivel de microcuencas, se concluyó la descripción textual del medio físico y demográfico, y se realizó el balance hidrológico superficial anual y mensual a nivel de microcuenca, así como el balance de aguas subterráneas.

La cartografía generada a escala 1:1 000 000, con una proyección cartográfica universal transversa de Mercator (UTM), y el sistema geodésico de referencia horizontal conocido como ITRF92 época 1980 cubren los temas: ubicación geográfica de la subcuenca, municipios que integran la subcuenca, población (localidades y número de habitantes), grado de marginación a nivel de localidad, relieve (altitud del terreno sobre el nivel medio del mar), subprovincias fisiográficas, sistemas de topoforma, precipitación total anual, temperatura media anual, unidades climáticas, geología (litología superficial, tipos de roca), suelos (unidades de suelo con sistema de clasificación FAO-CETENAL), cobertura del suelo, municipios, hidrografía, asentamientos humanos y número de habitantes.

Por otro lado, se delimitaron la cuenca y sus microcuencas.

\subsection{Etapa de educación}

En la etapa de educación, se comenzó con una sensibilización social a través de la presentación del cortometraje Saberes, en el cual se incluyeron las entrevistas con los adultos de la cuenca, quienes manifiestan en dicho material su saber histórico y sensibilizan a las nuevas generaciones 
respecto al cuidado de los recursos naturales para su beneficio y el de las próximas generaciones.

Esta elección metodológica logró una concientización en sus espectadores, y con ella un involucramiento de diferentes actores y líderes sociales, especialmente presidentes ejidales, quienes fungieron como representantes en la asociación civil que se formó.

La capacitación social se entendió en este proyecto como una forma del poder de la sociedad que la vincula con las políticas, los organismos y los programas sociales, y como una herramienta que permitió eliminar bloqueos al empoderamiento social al evitar la restricción de temas de debate, el ocultamiento de los conflictos, la salvaguarda de los intereses de grupos, la perpetuación del statu quo de la representatividad, la limitación de los recursos, la utilización de sistemas y lenguajes de participación limitativos, la obstaculización de la participación de grupos o individuos interesados, y la exclusión de posiciones personales o colectivas.

Por tanto, se hizo una integración horizontal de la comunicación y de la cooperación, fortaleciendo el capital social de normas organizaciones y las redes que permiten la toma de decisiones ciudadanas, y que se traducirán en políticas públicas y programas locales dictados desde el seno de la sociedad a través del ordenamiento ecológico territorial participativo.

La estrategia de desarrollo de capacidades sociales de autogestión se centró en el Comité de Cuenca de la Laguna de Santiaguillo, pero impactó en la comunidad de la cuenca debido a que el comité se compone de representantes de todos los sectores. La implantación generó un debate social de las posiciones y opiniones acerca de la problemática ambiental a resolver. 


\subsection{Etapa de acción}

Se logró la conformación del Comité de Cuenca de la Laguna de Santiaguillo de una forma democrática y representativa, lo que permite que se encuentre legitimado por los representantes sociales de los 30000 habitantes de las ciento cincuenta y una localidades. Este comité se denominó oficialmente Comité Activo de la Cuenca de Santiaguillo A. C. (CACUSAC), elaboró y validó su reglamento interno, y acordó su agenda de sesiones y su plan general de trabajo. El comité se integró de manera formal ante notario público con representantes de cada microcuenca.

La asociación civil detonó varios procesos de capacitación para la autogestión, incluyendo desde aspectos técnicos hasta organizativos, y mostrando un gran poder de convocatoria en las comunidades que integran la cuenca. También realizó, mediante el apoyo de los técnicos capacitados de la zona de estudio dentro del proyecto, la cartografía integrada con todos los temas, misma que luego socializó, apropiándose con ello del concepto de cuenca hidrológica. Igualmente, se vinculó activamente con los proyectos de investigación y tesis de posgrado, que le han ido generando información para enriquecer sus diagnósticos.

Una de las primeras manifestaciones de acción del comité fue la organización de una exposición itinerante de mapas por los distintos ejidos de la cuenca, en la cual dichos mapas se presentan en cada una de las reuniones ejidales y son explicados por los propios habitantes de la cuenca que tomaron el curso de sistemas de información geográfica, esto con el fin de dar a conocer a los pobladores de la cuenca cómo se conforma el territorio y sensibilizarlos con relación a su cuidado. Esta estrategia permitió posicionar el concepto de cuenca hidrográfica en los habitantes.

Además, la asociación civil gestionó y logró la firma del ordenamiento intermunicipal de la cuenca de Santiaguillo, del cual forma parte como consejera en tanto representante 
de la sociedad civil que habita en la cuenca. Su responsabilidad en dicho ordenamiento es buscar el bien común y la acción colectiva de todos los involucrados para el manejo integral y sustentable de la cuenca. El ordenamiento está integrado por los tres niveles de Gobierno, instituciones de investigación como el Instituto Politécnico Nacional (IPN), representantes de la sociedad civil como el CACUSAC, y otros actores relevantes para la toma de decisiones relacionadas con el manejo sustentable de la cuenca.

\section{4. Etapa de cierre}

La asociación civil CACUSAC sesiona ahora de forma autónoma, y el papel que juega el IPN es ser parte de su red de apoyo, lo que fortalece el proceso de autogestión. Ha reemplazado de forma rápida y eficiente las deserciones internas, que se deben a diversas razones, y ha manteniendo la representatividad de la cuenca. De igual modo, soluciona de forma eficaz y eficiente los conflictos internos que se presentan, manteniendo una unión de los socios. Hoy día, el CACUSAC comienza a incidir en la vida social, productiva y económica de la cuenca mediante acciones de impacto profundo en la toma de decisiones de tipo ambiental, ya sea en acuerdo con las autoridades gubernamentales o de forma independiente.

También, la asociación civil realiza diagnósticos de la condición ambiental, social y de la gestión con el apoyo y la asesoría del IPN y de algunas otras asociaciones civiles, como Pronatura Noreste A. C. Es decir, el comité de la cuenca es ahora un organismo de la sociedad que comienza a ganar reconocimiento y cuenta ya con la validación necesaria para proponer e integrar las distintas alternativas de gestión de los recursos naturales y preservación ambiental que realizan, por ahora de forma dispersa, los sectores comunitarios, políticos y privados. 


\section{Conclusiones}

La metodología de la IAP empleada en el caso de estudio señalado en este trabajo ha permitido la creación de un grupo autogestivo (asociación civil) que, aunque se encuentra en fase de consolidación y capacitación, comienza a mostrar posibilidad de sobrevivencia ahora que el IPN ya se ha retirado de la zona de estudio.

Con ello, se ha sembrado en los habitantes de la zona una visión diferente de la manera de atender las demandas que genera el entorno ambiental sobre los sectores productivo, social y económico, a fin de asegurar la sustentabilidad.

La Comisión Nacional de Áreas Naturales Protegidas inició en 2015 la elaboración de un plan de gestión del sitio Ramsar que se encuentra en la Laguna de Santiaguillo, y ha solicitado la intervención del CACUSAC como representante de la sociedad de la cuenca. También la Secretaría de Recursos Naturales (SEMARNAT), por su parte, reconoció a la asociación como representante de la sociedad civil de la cuenca, por lo que, como se dijo antes, la integró como parte del consejo para el ordenamiento intermunicipal de la misma.

Se considera que la gobernanza ambiental es un proceso que implica la cooperación, la acción colectiva y la voluntad de muchos actores públicos y privados para alcanzar propósitos comunes que encaminen los esfuerzos hacia el uso sustentable de los recursos hídricos, siendo eje importante de la misma la participación ciudadana. Implica liderazgos fuertes y comprometidos, además de una serie de acciones e instituciones que deben acompañar a las asociaciones civiles para su conformación y fortalecimiento con asesoría, diagnóstico, planeación estratégica y financiamiento, entre otras.

Se concluyó que una sociedad civil integrada por usuarios y líderes de una región a nivel cuenca es capaz de promover la gobernanza ambiental y encaminar acciones como un ordenamiento de cuenca para el uso sustentable de los 
recursos hídricos. También se concluyó que la participación de las autoridades está sujeta a la presión que ejerzan la propia ciudadanía e instituciones de investigación y no gubernamentales, produciendo una sinergia benéfica para la gestión ambiental.

Ávalos, H. C., Alcántar, A. G., Mora, I. D. G., López, R. F. P., y Patrón, E. R. (20I3). Cuencas hidrográficas. Fundamentos y perspectivas para su manejo y gestión. Cuadernos de divulgación ambiental. Guadalajara: Secretaría de Medio Ambiente y Recursos Naturales.

Balcazar, F. E. (2003). “Investigación acción participativa (IAP): aspectos conceptuales y dificultades de implementación". Fundamentos en humanidades, 7(8).

Comisión Mundial sobre el Medio Ambiente y el DesarroIlo (1987). Nuestro futuro común o Informe Brundtland. Brundtland: Organización de las Naciones Unidas. Recuperado de: https://es.scribd.com/doc/ /05305734/ ONU-Informe-Brundtland-Ago-1 987-Informe-de-laComision-Mundial-sobre-Medio-Ambiente-y-Desarrollo Costanza, R., Cumberland, J., Daly, H., Goodland, R., y Norgaard, R. (1999). Una introducción a la economía ecológica. México: AENOR.

Cotler, H. (2007). “Introducción a la segunda edición”, en H. Cotler (Comp.), El manejo integral de cuencas en México: estudios y reflexiones para orientar la política ambiental (PP. II-17). México: SEMARNAT.

Delgado, L., Bachmann, P., y Oñate, B. (2007). “Gobernanza ambiental: una estrategia orientada al desarrollo sustentable local a través de la participación ciudadana”. Revista ambiente y desarrollo de CIPMA, 23(3), 68-73.

Donzier,J. F.,Walshe, M., Brühl, H., Cordeiro, O. D. M., Estrela, T., et al.(2009). Manual para la gestión integrada de recursos hídricos en cuencas. Francia: Global Water Partnership, International Network of Basin Organizatinons. 
Dúran, S. (2009). "El tercer sector en la cultura: redes, asociaBibliografía ciones, organizaciones". Red Iberoamericana de Ciudades para la Cultura, 13, s/p.

Espinoza, L. E. (2004). “Una visión critica sobre el 'desarollo sostenible”, en J.M. García, y C.Velayos (Coords.), Tomarse en serio la naturaleza: ética ambiental en perspectiva multidisciplinar (pp. 289-317). España: Biblioteca Nueva.

García, P. (2008). "Organizaciones del tercer sector: análisis general de la publicidad de la "“Fundación UNAM, A. C.”. Espacios públicos, II(2I), I8.

Granovetter, M.(1978).“'Threshold Models of Collective Behavior”. The American Journal of Sociology, 83(6), I420-I 443.

Hardin, G. (1968). "The Tragedy of Commons”. Science, I62, I243-। 248.

Jiménez, L. (2003). "Cambio global, desarrollo sostenible y economía ecológica”, en L. Jiménez, y F. Higón (Coords.), Ecología y economía para un desarrollo sostenible (pp. 13-62). Valencia: Universitat dValencia.

Kirchner, A. (2007). La investigación acción participativa (IAP). Argentina: Foro Latinoamérica .

Kooiman, J. (2004). “Gobernar en gobernanza”. Instituciones y desarrollo, 16, I7I-194.

Maass, M., y Cotler, H. (2007). “El protocolo para el manejo de ecosistemas en cuencas hidrográficas", en $\mathrm{H}$. Cotler (Comp.), El manejo integral de cuencas en México (pp. 4I-58). México: SEMARNAT.

Montenegro, M. O. G. (20I4). Análisis de los factores naturales y antropógenicos que inciden en la disponibilidad de agua en la cuenca de la Laguna de Santiaguillo. Durango: Instituto Politécnico Nacional.

Moya, L. M. M. (2004). "Acción colectiva y modelos de racionalidad". Estudios fronterizos, 5(9), 25.

Nowak, M. (2006). "Five rules for the evolution of cooperation". Science, 3/4(5805), 12. 
Bibliografía
Ostrom, E. (2000). “El gobierno de los bienes comunes. La evolución de las instituciones de accion colectiva”. Región y sociedad, XIV, 395.

Pereda, C.,Prada, M.A.,yActis,W.(1993).“'Investigación acción participativa: introducción en España”. Documentación social, 92, 59-70.

Piedra, J. I. G. (2007). “El manejo de cuencas en Cuba”, en H. Cotler (Comp.), El manejo integral de cuencas en México: estudios y reflexiones para orientar la política ambiental (pp. 2I-40). México: SEMARNAT.

Vargas, J. G. (2003). "Teoría de la acción colectiva, sociedad civil y los nuevos movimientos sociales en las nuevas formas de gobernabilidad en Latinoamérica”. Espacio abierto, 12(4), 16.

Vélez, G.S.(20I I).Economía ecológica:las pequeñas empresas y el desarrollo sustentable. Dilemas del mercado global, I ( I), I07.

Zárate, G. C. (2007).“Del gobierno a la gobernabilidad de los recursos hídricos en México”, en H. Cotler (Comp.), El manejo integral de cuencas en México: estudios y reflexiones para orientar la política ambiental (pp. 173-182). México: SEMARNAT. 